\title{
Proximal radial pseudotumour: magnetic resonance imaging appearances and prevalence
}

\section{Ian Pressney*}

Department of Radiology, Royal National Orthopaedic Hospital, Brockley Hill, Stanmore, Middlesex, HA7 4LP, UK

Received: 05 February, 2020

Accepted: 14 May, 2020

Published: 15 May, 2020

*Corresponding author: lan Pressney, Department of Radiology, Royal National Orthopaedic Hospital, Brockley Hill, Stanmore, Middlesex, HA7 4LP, UK, E-mail: ipressney@hotmail.com

Keywords: Pseudo tumour; Radial tuberosity; Red marrow; Magnetic resonance imaging

https://www.peertechz.com

Check for updates

\begin{abstract}
Introduction: Cases have been referred to our tertiary referral sarcoma service where marrow abnormalities have been identified in the proximal radius. We believe this to be a normal variant, likely representing residual red marrow. The purpose of this study is to describe the features and determine the prevalence of such a finding on elbow magnetic resonance imaging (MRI) in an unselected group of patients imaged at our institution.

Materials and methods: Retrospective review of the last 100 elbow MRI studies performed at our institution. Patient age, sex, side imaged and reason for MRI were recorded. The proximal radial pseudotumour, was defined as a region of marrow signal abnormality with intermediate T1W SE/ T2W FSE/PDW FSE signal intensity between medullary fat and skeletal muscle with hyperintense STIR/PDW FS signal compared to medullary fat, but hypointense to joint fluid. For positive cases, the length and distance from the proximal radial articular surface was measured. Also, local changes, including cortical abnormality, active periostitis, surrounding soft tissue abnormality and similar change in distal humerus and proximal ulna were assessed.
\end{abstract}

Results: The pseudotumour was identified in $7.8 \%$ of cases. No statistical differences were observed for age $(p=0.1)$, laterality $(p=0.08)$ or sex $(p=0.79)$ for pseudotumour presence. Mean length of pseudotumour was $21 \mathrm{~mm}$ with mean distance from the articular surface of $16 \mathrm{~mm}$.

Conclusions: The proximal radial pseudotumour is seen in $7.8 \%$ of elbow MRI studies and should be recognised as a normal variant of proximal radial marrow signal intensity.

\section{Introduction}

A pseudo-tumour could be defined as an abnormality that resembles a tumour but with an absence of neoplastic cells. Many pseudo-tumours have been described in the radiology literature [1], some of which relate to marrow signal abnormalities that present as potential interpretational pitfalls. Marrow signal abnormalities are becoming increasingly frequent dilemmas given the burgeoning use of fat suppressed imaging. The bone marrow is a complex organ whose proportions can vary greatly depending upon site and age [2,3], with typical conversion of yellow to red marrow in long bones occurring from the end of the bone to the central diaphyseal region [4]. Some studies have demonstrated that variations in this process exist [5]. However, islands of red marrow can persist from the original conversion stage into adulthood [3]. Multiple studies have been conducted looking at red marrow MRI signal around the knee joint in an attempt to assess its frequency and its clinical significance [68]. The presence of this red marrow has been assessed against patient factors such as weight, age, sex, haemoglobin levels and smoking. The elbow is a less frequently imaged joint than the knee but there have been observations with regards to alteration of marrow signal around the elbow, particularly in the distal humerus [9]. The purpose of the current study was to assess the incidence of this imaging feature at the elbow joint, particularly within the proximal radius following several cases referred to our institution with potential diagnoses of occult fracture, stress injury, reactive marrow oedema, osteitis and osteomyelitis.

\section{Materials and methods}

Following local Research and Development Board approval, a retrospective study of the most recent 100 consecutive MRI studies of the elbow joint was undertaken. MRI studies from both 1.5 and 3 Tesla (T) units were included in the study. 
Patient's age, sex, side and reason for MRI were documented. Clinical indications for elbow imaging were divided into 3 groups. Group $1(n=43)$ : patients referred for a potential mass lesion or lesion follow-up of a mass; Group $2(n=13)$ : patients referred for assessment of ulnar nerve and transpositions; Group $3(n=34)$ : patients referred for investigation of a variety of elbow abnormalities including trauma, dislocation, congenital abnormality, soft tissue injuries, including ligamentous and joint degeneration.

The presence of a pseudo-tumour in the proximal radius was assessed by 2 consultant musculoskeletal radiologists with 4 and 7 years of experience, with the result agreed by consensus.

A radial pseudo-tumour was defined as a focal region of marrow abnormality in the proximal radius which had intermediate signal intensity (SI) between medullary fat and skeletal muscle on T1-weighted spin echo (W SE), T2-weighted turbo spin echo (W TSE) and proton density weighted turbo spin echo (PDW TSE) sequences, and was hyperintense to medullary fat but hypointense to joint fluid on short tau inversion recovery (STIR) and spectral attenuated inversion recovery (SPAIR) sequences. If the study was positive for a pseudo-tumour, then the dimensions of the lesion were measured as well as its distance from the proximal radial articular surface. If positive, then other local changes including cortical abnormality, active periostitis, surrounding soft tissue abnormality including oedema were recorded. Assessment for similar SI change in the distal humeral metaphysis and proximal ulna was also undertaken.

MRI parameters included 1.5T and 3T 'tumour' and 'nontumour' protocols, as follows

1. $1.5 \mathrm{~T}$ (Philips Achieva) 'tumour' protocol; coronal T1W SE (TR 579.84, TE 20) and STIR (TR 4278.44, TE 30, TI 190), axial PDW FSE (TR 4502.4, TE 25) and SPAIR (TR 3211.4, TE 30), and sagittal T2W TSE (TR 3000, TE 90) sequences. Field of view (FOV): $120 \times 120 \mathrm{~mm}$, slice thickness (ST): 3mm, 2 channel elbow coil.

2. 'Non-tumour' 1.5T protocol; coronal PDW FSE (TR 3500, TE 30) and SPAIR (TR 3653.5, TE 30), axial PDW FSE (TR 3000, TE 30) and SPAIR (TR 5561.3, TE 30), and sagittal T2W fast field echo (FFE) (TR 550, TE 11.5) sequences. FOV: $120 \times 120 \mathrm{~mm}, \mathrm{ST}: 3 \mathrm{~mm}, 2$ channel elbow coil.

3. 3T (Philips Ingenia) 'tumour' protocol; coronal $\mathrm{T} 1 \mathrm{~W}$ SE (TR 526.96, TE 20) and STIR (TR 4465.31, TE 60, TI 210), axial PDW FSE (TR 3000, TE 30) and SPAIR (TR 3782.45, TE 30), and sagittal T2W TSE (TR 1934.68, TE $80)$ sequences. FOV: $120 \times 120 \mathrm{~mm}$, ST: $3 \mathrm{~mm}, 6$ channel elbow coil.

4. 'Non-tumour' 3T protocol; coronal PDW FSE (TR 3126.19, TE 30) and SPAIR (TR 3059.09, TE 30), axial PDW FSE (TR 3000, TE 30) and SPAIR (TR 3057.71, TE 30), and sagittal T2W TSE (TR 1947.02, TE 80) sequences. FOV: 120x120mm, ST: 3mm (axial 2.5mm), 6 channel elbow coil.

Studies were excluded if the proximal radius was not included in the imaged field $(n=3)$, if marrow signal was obscured by metal artifact $(n=3)$ or if the proximal radius was involved by any pathological process involving the marrow $(n=4)$. Four cases had pathology at the radial tuberosity affecting the adjacent marrow as follows; cystic change underlying the cortex of the radial tuberosity secondary to distal biceps tendinosis, cortical erosion and marrow-oedema-like SI in the radial tuberosity secondary to bicipitoradial bursitis, two cases of neoplastic marrow infiltration due to histologically proven leiomyosarcoma, and multiple lesions consistent with Brown tumours in patient with known hyperparathyroidism.

Univariate analysis to assess for association between age, sex, side and the presence of a pseudo-tumour was undertaken. A p-value of $<0.05$ was taken as statistically significant.

\section{Results}

Of the 100 successive elbow MRI cases identified, 10 were excluded. Therefore, the final study group comprised of 90 patients, 43 males and 47 females with a mean age of 41 years and age range of 7-80 years.

There were $11(12.2 \%)$ patients imaged at $1.5 \mathrm{~T}$ and 79 $(87.8 \%)$ at $3 \mathrm{~T}$. Proximal radial pseudo-tumours were identified in $7(7.8 \%)$ patients (Figures $1-5), 3$ males and 4 females ( $p=$ 0.79). Pseudo-tumours involved the right elbow in 6 cases and left elbow in $1(p=0.08)$. Age range for pseudo-tumour was 7-65 years with mean age 30 years, compared with 5-80 years with mean 42 years for no pseudo-tumour $(p=0.1)$. The pseudotumours measures between 5 to $38 \mathrm{~mm}$ in length with a mean of $21 \mathrm{~mm}$, and the distance from the radial articular surface varied from 4 to $32 \mathrm{~mm}$ with a mean of $16 \mathrm{~mm}$. None of the pseudotumours was associated with cortical abnormality, active periostitis or soft tissue oedema-like SI. No abnormal marrow SI was identified in the distal humeral metaphysis or proximal ulna on any of the imaging series.

\section{Discussion}

Bone marrow is regarded as a dynamic organ and undergoes lifelong changes, with the capability to reverse these changes when placed under certain stresses [10]. It is this ability that renders bone marrow susceptible to changes as a result of not only metabolic stimulation, but also hyperactive osteoclastic activity. This can result in a pseudo-tumour appearance as
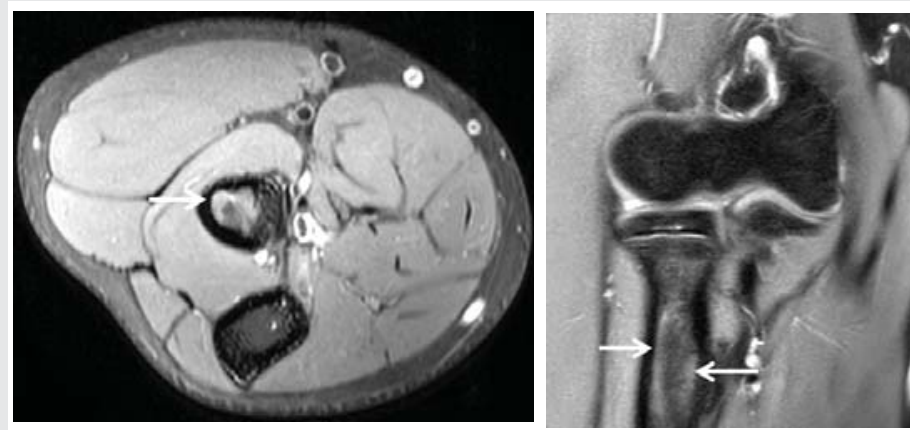

Figure 1: Axial PDW SPAIR (TR 3527.81; TE 30) and b coronal PDW SPAIR (TR 3238.66; TE 30) in 14 year old year old female referred for potential fracture demonstrates a pseudo tumour in radial tuberosity (white arrows). Compare with the marrow signal in adjacent proximal ulna (Figure 1a). 
something that mimics malignancy or an aggressive process. Reconversion of yellow to red marrow is considered to occur in an opposite longitudinal direction to its initial conversion, with reconversion widely accepted to begin in the ends of long bones then extending to the middle or central diaphyseal regions [4]. It is also accepted that there may be residual red marrow at the last conversion sites, which are in the proximal metaphyses of the femur and humerus. It is well-documented that these residual red marrow foci can show wide variation in location and morphology across the ages at these sites [3], but there is little literature on red marrow at sites other than these, such as around the elbow joint. This rarity of marrow signal abnormality in and

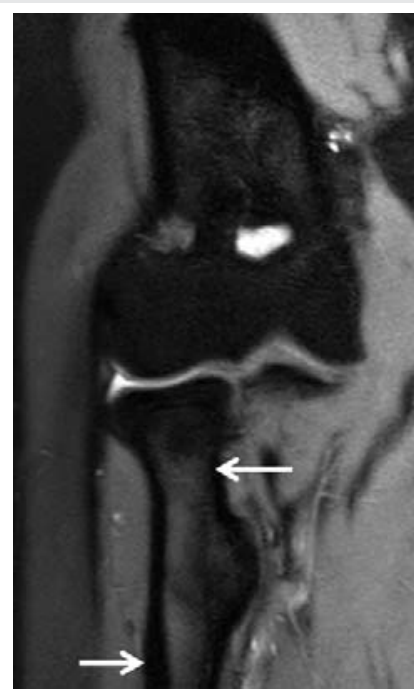

Figure 2: Coronal PDW SPAIR (TR 3885.5; TE 30) in 15 year old year old female referred for potential osteochondral defect demonstrates a pseudo tumour in radial tuberosity.
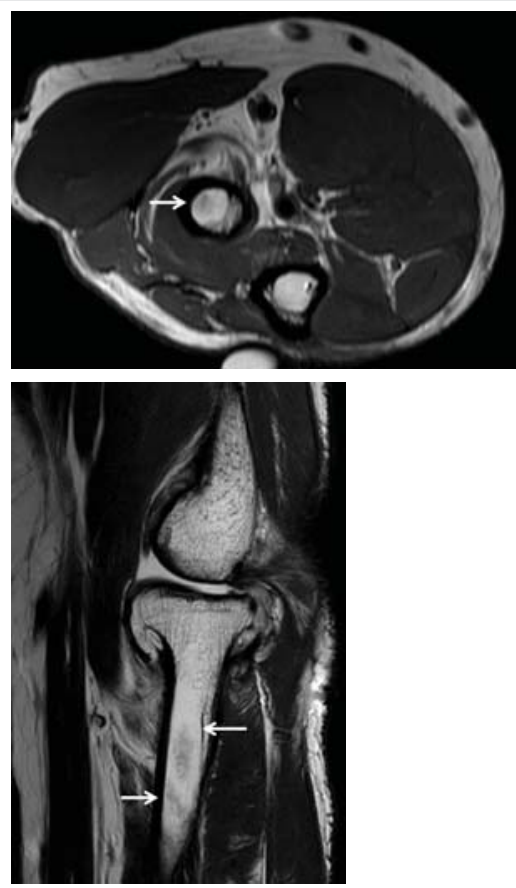

Figure 3: a. Axial PDW TSE (TR 4142.95; TE 30) and sagittal T2W TSE (TR 2421.35; 80 ) in 54 year old year old male referred for potential occult fracture demonstrates a pseudo tumour in radial tuberosity (white arrows). Compare with the marrow signal in adjacent proximal ulna (Figure 3a).

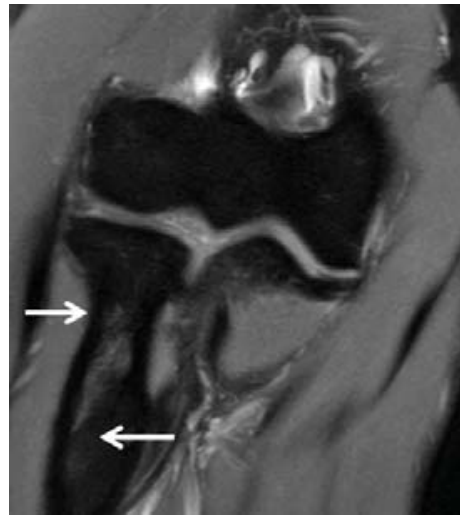

Figure 4: Coronal PDW SPAIR (TR 3885.59; TE 30) in 36 year old male referred for potential occult fracture demonstrates a pseudo tumour in radial tuberosity.c
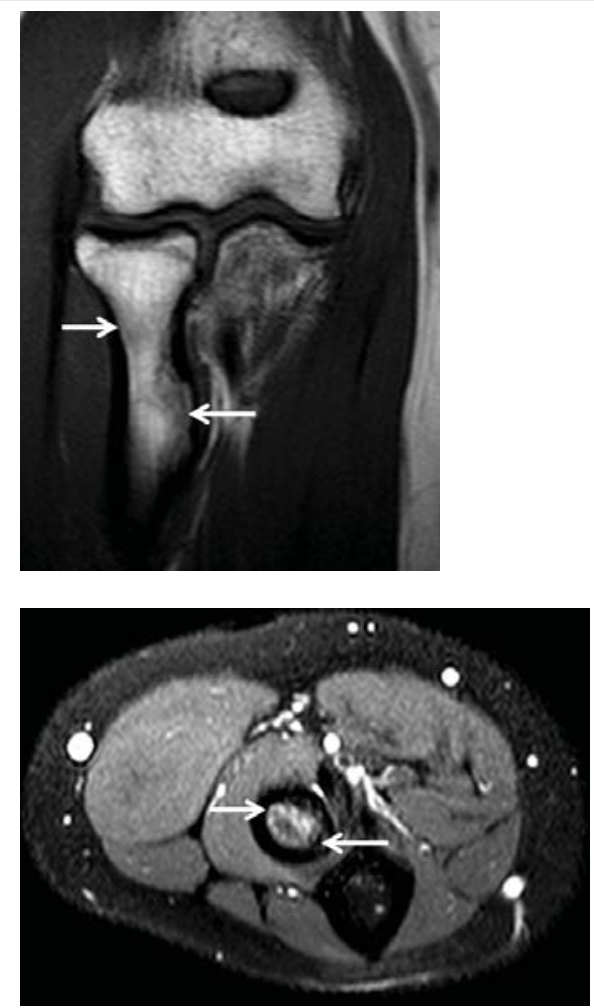

Figure 5: a. Coronal T1W TSE (TR 500; TE 15) and b axial PDFS (TR 4451.33; TE 30) in 15 year old year old female referred for mass in proximal forearm demonstrates a pseudo tumour in radial tuberosity (white arrows). Compare with the marrow signal in adjacent proximal ulna (Figure $5 \mathrm{~b}$ ).

around the elbow joint is confirmed in a study that had only 1 elbow MRI study with a report of incidentally noted 'abnormal or heterogenous marrow signal' out of a total of 49,678 MRI studies [11]. A more recent study suggested wide variation in red marrow signal with diaphyseal marrow reconversion appearing to precede that of distal metaphyseal marrow in the femur [5]. Focal nodular hyperplasia of red marrow has been described as an abundance of focal red marrow due to aberrant red to yellow marrow conversion [12]. It is described as a rarely occurring localised form in the marrow of the spine and pelvis, compared to regional forms around the knees of endurance athletes, heavy smokers and in obesity [13]. Therefore, it is important to recognise aberrant marrow signal intensity as highlighted in our current study, and we postulate that this proximal radial 
pseudo-tumour represents a normal variant representing residual haemopoietic red marrow with morphology like that of focal nodular hyperplasia.

This is supported by various authors who have researched red marrow signal in and around the knee. Wilson, et al. [7], found clear gender, age, obesity and smoking-related differences in the prevalence of red marrow around the knee joint with a prevalence of $35 \%$. They concluded that its presence should not be a cause for clinical concern. In another study, Haemoglobin $\mathrm{Hb}$ ) levels were not found to have an effect on marrow signal in the distal femoral metaphysis [6], although a later study suggested that complete marrow replacement in the distal femoral metaphysis in women was associated with significantly lower $\mathrm{Hb}$ levels, prompting advice of checking $\mathrm{Hb}$ levels if incidentally found on MRI [8]. Haemopoietic marrow has also been documented as having high prevalence in asymptomatic marathon runners [14].

The current study uses basic MRI sequencing to assess the marrow signal which is universally applied to most MRI protocols for assessment of marrow lesions and its characteristics. It utilises the inherent maintenance of intrinsic fat content within the red marrow resulting in intermediate SI between medullary fat and skeletal muscle on T1-W SE and T2-W TSE. Advances in MRI techniques have led to new sequences as problemsolving tools in the assessment of marrow lesions that have the potential benefit of quantification. This includes the DIXON technique which relies on the resonance frequencies of fat and water resulting in the ability to quantify the amount of fat within a bone lesion [15]. Established 'cut-off' threshold levels of $<20 \%$ (1.5Tesla) or $<25 \%$ (3Tesla) signal intensity decrease has high sensitivity and specificity for a non-neoplastic lesion such as hyperplastic red marrow. [15]. Historically, the quantifiable DIXON technique has utilised T1-W imaging, but T2-W DIXON has recently been shown to adequately differentiate between yellow, red marrow and osseous lesions [16]. Diffusion weighted (DW) imaging is a different type of quantifiable MRI sequence that utilises measuring Brownian motion of water molecules in a voxel [17]. More recently intravoxel-incoherent DW MRI demonstrated significantly lower pure diffusion coefficient values for hyperplastic marrow compared with vertebral malignant lesions, and outperformed Attenuated Diffusion Coefficient (ADC) for differentiating these lesions [18]. Novel superposition of $\mathrm{T} 1$ and $\mathrm{T} 2 \mathrm{~W}$ MRI with mathematical statistical dominance algorithms has produced reproducible results for growth zone delineation in adolescent wrists, prompting the authors to postulate that this could potentially add value in marrow lesion assessment [19].

An obvious limitation of our study was not obtaining information regarding other parameters, particularly weight, $\mathrm{Hb}$ levels, exercise levels and smoking history from the retrospective review, but the purpose of the study was to highlight the prevalence of this imaging feature in a routine and unselected group of patients undergoing elbow MRI.

\section{Conclusion}

The proximal radial pseudo-tumour is seen in approximately
$8 \%$ of elbow MRI studies, and should be recognised as a variant of proximal radial marrow signal. The increasing use of MRI will undoubtedly result in more incidental findings and documentation of the incidence and basic MRI imaging features at this location should increase confidence in MRI assessment and reduce the requirement to undertake needle biopsy for tissue diagnosis in this non-neoplastic condition.

\section{References}

1. Stacy GS, Dixon LB (2007) Pitfalls in $\mathrm{mr}$ image interpretation prompting referrals to an orthopaedic oncology clinic. Radiographics 27: 805-826. Link: https://bit.ly/2yUGEI1

2. Vande Berg BC, Malghem J, Lecouvet FE, Maldague B (1998) Magnetic resonance imaging of normal bone marrow. Eur radiol 8: 1327-1334. Link: https://bit.ly/2Lsys4k

3. Richardson ML, Patten RM (1994) Age-related changes in marrow distribution in the shoulder: MR imaging findings. Radiology 192: 209-215. Link https://bit.ly/2LrnGvr

4. Kricun M (1985) Red-yellow marrow conversion: its effect on the location of some solitary bone lesions. Skeletal Radiol 14: 10-19. Link: https://bit.ly/3bssxaf

5. Kim SC, Krynyckyi BR, Machoc J, Kim CK (2006) Patterns of red marrow in the adult femur. Clin Nucl Med 31: 739-741. Link: https://bit.ly/2Lr7vOx

6. Arslan G, Ozmen E, Soyturk M (2015) MRI of residual bone marrow in the distal femur of healthy subjects. Pol J Radiol 80:300-304. Link: https://bit.ly/2T23ios

7. Wilson AJ, Hodge JC, Pilgram TK, Hang EH, Murphy WA (1996) Prevalence of red marrow around the knee joint in adults as demonstrated on magnetic resonance imaging. Acad Radiol 3: 550-555. Link: https://bit.ly/2Wqynoj

8. Gonzalez FM, Mitchell J, Monfred E, Angub T, Mulligan M (2016) Knee MRI patterns of bone marrow reconversion and relationship to anaemia, Acta Radiol 57: 964-970. Link: https://bit.ly/3bsH6KP

9. Sampaio ML, Schweitzer ME (2010) Elbow magnetic resonance imaging variants and pitfalls. Magnetic Resonance Imaging Clinics of North America 18: 633-642. Link: https://bit.ly/3fM40jS

10. Gurevitch O, Slavin S, Feldman AG (2007) Conversion of red bone marrow into yellow- cause and mechanisms. Med Hypotheses 69: 531-536. Link: https://bit.ly/3cFiQGE

11. Shah GL, Rosenberg AS, Jarboe J, Klein A, Cossor F (2014) Incidence and evaluation of incidental abnormal bone marrow signal on magnetic resonance imaging. Scientific World Journal 2014: 380814. Link: https://bit.ly/3cwv3NS

12. Bordalo-Rodrigues M, Galant C, Lonneux M, Clause D, Vande Berg BC (2003) Focal nodular hyperplasia of the hematopoietic marrow simulating vertebra metastasis on FDG positron emission tomography. Am J Roentgenol 180 669-671. Link: https://bit.ly/2Ws00C

13. Poulton TB, Murphy WD, Duerk JL, Chapek CC, Feiglin DH (1993) Bone marrow reconversion in adults who are smokers: MR imaging findings. AM J Roentgenol 161: 1217-1221. Link: https://bit.ly/3dETUPS

14. Shellock FG, Morris E, Deutsch AL, Mink JH, Kerr R, et al. (1992) Hematopoietic bone marrow hyperplasia: high prevalence on MR images of the knee in asymptomatic marathon runners. AJR Am J Roentgenol 158: 335-338. Link: https://bit.ly/2T4s0Fc

15. van Vucht N, Santiago R, Lottmann B, Pressney I, Harder D, et al. (2019) The Dixon technique for MRI of the bone marrow. Skeletal Radiol 48: 1861-1874 Link: https://bit.ly/2AglAuL 
16. Sasiponganan C, Yan K, Pezeshk P, Xi Y, Chhabra A (2019) Advanced MR imaging of bone marrow: quantification of signal alterations on T1weighted Dixon and T2-weighted Dixon sequences in red marrow, yellow marrow, and pathologic marrow lesions. Skeletal Radiol. 49: 541-548. Link: https://bit.ly/3fIWZQI

17. Khoo M, Tyler PA, Saifuddin A, Padhani AR (2011) Diffusion-weighted imaging (DWI) in musculoskeletal MRI: a critical review. Skeletal Radiol 40: 665-681. Link: https://bit.ly/3cvSbw3
18. Park S, Kwack KS, Chung NS, Hwang J, Lee HY, et al. (2017) Intravoxe incoherent motion diffusion-weighted magnetic resonance imaging of focal vertebral bone marrow lesions: initial experience of the differentiation of nodular hyperplastic hematopoietic bone marrow from malignant lesions. Skeletal Radiol 46: 675-683. Link: https://bit.ly/3dHNobe

19. Obuchowicz R, Urbanik A, Piórkowski A (2019) Novel Technique for Growth Plate Analysis Based on the Superposition of T1- and T2weighted MR Imaging of Adolescent Wrists. Magn Reson Med Sci. Link: https://bit.ly/2zCfupC
Discover a bigger Impact and Visibility of your article publication with Peertechz Publications

\section{Highlights}

* Signatory publisher of ORCID

* Signatory Publisher of DORA (San Francisco Declaration on Research Assessment)

* Articles archived in worlds' renowned service providers such as Portico, CNKI, AGRIS, TDNet, Base (Bielefeld University Library), CrossRef, Scilit, J-Gate etc.

* Journals indexed in ICMJE, SHERPA/ROMEO, Google Scholar etc.

- OAI-PMH (Open Archives Initiative Protocol for Metadata Harvesting)

* Dedicated Editorial Board for every journal

- Accurate and rapid peer-review process

* Increased citations of published articles through promotions

* Reduced timeline for article publication

Submit your articles and experience a new surge in publication services (https://www.peertechz.com/submission).

Peertechz journals wishes everlasting success in your every endeavours.

Copyright: ( $) 2020$ Pressney I. This is an open-access article distributed under the terms of the Creative Commons Attribution License, which permits unrestricted use, distribution, and $r$ eproduction in any medium, provided the original author and source are credited. 